\title{
Investigations on the stratification dynamics and the gas concentrations of Lake Kivu
}

\author{
MAXIMILIAN SCHMIDT ${ }^{1}$, DAVID WACHS ${ }^{2}$, YANNIS \\ WOLFGANG ARCK ${ }^{3}$, FABIAN BÄRENBOLD ${ }^{4}$, LISA \\ RINGENA $^{5}$, JULIAN ROBERTZ ${ }^{5}$, BERTRAM BOEHRER ${ }^{6}$, \\ MARKUS K OBERTHALER ${ }^{5}$ AND WERNER AESCHBACH ${ }^{2}$ \\ ${ }^{1}$ Institute for Environmental Physics Heidelberg \\ ${ }^{2}$ Institute of Environmental Physics \\ ${ }^{3}$ Institute for Environmental Physics \\ ${ }^{4}$ Eawag \\ ${ }^{5}$ Kirchhoff-Institute for Physics \\ ${ }^{6}$ Helmholtz Zentrum für Umweltforschung UFZ Magdeburg \\ Presenting Author: schmidtmags@googlemail.com
}

Lake Kivu, a meromictic lake in the East African Great Rift Valley, is characterized by its very stable salt driven stratification and its high volcanogenic gas concentration. The constant gas input ( $\mathrm{CO} 2$ and $\mathrm{CH} 4)$ in combination with the stable water column might lead to disastrous degassing events, limnic eruptions, as happened before at the lakes Nyos and Monoun in Cameroon. To get insights to the water column dynamics and thus historic eruptions our aim is to date the age of the water at different depth using 39Ar Atom Trap Trace Analysis, short ArTTA.

The noble gas isotope ${ }^{39} \mathrm{Ar}\left(\mathrm{t}_{1 / 2}=269\right.$ a) covers a unique time span for studying the dynamics of aquatic and glacial systems of the last millennium. Although this tracer has been acknowledged for decades, studies so far are limited by its low abundance, little radioactivity and hence huge required sample sizes $(\sim 1000 \mathrm{~L}$ water). Until today environmental routine measurements are mainly confined to groundwater reservoirs, where nearly unlimited sampling is possible. The application of techniques from atomic physics using a magneto optical atom trap (MOT) solves the problem by reducing sample volume requirements by several orders of magnitude. The problem of the very low isotopic abundance of $10^{-16}$ is resolved by resonant multi-photon scattering of ${ }^{39} \mathrm{Ar}$ in the MOT. This technique named Argon Trap Trace Analysis with its very low minimal sample size of 0.5 $\mathrm{cm}^{3}$ STP pure argon enables easy sample handling in the field as well as common sampling procedures like Niskin bottles for aquatic systems, drill core sampling for glacial systems or as in the case of Lake Kivu spray chamber gas sampling in remote places. It is thus a door opener for new geophysical research fields that were excluded from radio-argon dating so far.

Beside dating ${ }^{39} \mathrm{Ar}$ dating, other efforts have been undertaken to investigate Lake Kivu's gas content and composition utilizing a total dissolved gas probe beyond its actual capabilities.

We here want to present our most recent results from dating Lake Kivu with ArTTA as well as some insights on how to expand the capabilities of a total dissolved gas probe. 